\title{
Parenting a child with haemophilia while living in a non-metropolitan area
}

Trudi Little, Esben Strodl*, Simon Brown, Tara Mooney

The experience of living in a non-metropolitan area and parenting a child with haemophilia is relatively unknown. Using Interpretive Phenomenological Analysis (IPA), the following study explored the experiences of seven parents, from which four themes emerged: 'bearing the brunt of diagnosis' captures the impact of the diagnosis; 'if you can't help me, who can?' reveals experiences with the health system; 'tackling the challenge of treatment' encompasses difficulties in adhering to the treatment regime; 'I need you to understand' reflects desires for others support and understanding. These themes should be considered when developing support systems and interventions for parents living in non-metropolitan areas.

Keywords: haemophilia, parents, interpretative phenomenological analysis

Severe haemophilia is usually diagnosed within the first year of life [1]. Previously, children with severe haemophilia were rarely expected to survive beyond their first decade of life and quality of life was extremely poor [2]. However, contemporary treatment options have allowed for better management and prevention of severe bleeds, and children with haemophilia can now lead relatively normal and healthy lives $[1,3]$. The treatment of haemophilia involves the injection of clotting factor concentrate into a vein or implantable central venous line in order for blood to clot normally.

Prophylaxis is the primary treatment for severe haemophilia in Australia [1,4]. Parents administer treatment up to four times per week in order to prevent spontaneous, muscularskeletal bleeds, which cause severe pain and swelling of joints, and can be a significant risk to life $[1,3,4]$. Around $4 \%$ of males with haemophilia will experience life-threatening, intracranial haemorrhages during the neonatal period [5]. Without treatment, individuals are at risk of incapacitation by progressive and severe chronic arthropathy developed from repeated joint bleeds, leading to chronic pain, decreased functionality and poor quality of life [4]. Hence there is considerable pressure on parents to be intimately involved

Dr Esben Strodl, School of Psychology and Counselling, Queensland University of Technology, Victoria Park Road, Kelvin Grove, Queensland, 4059, Australia Email: e.strodlaqut.edu.au

Trudi Little, Queensland University of Technology, Brisbane, Australia Simon Brown, Lady Cilento Children's Hospital, Brisbane, Australia Tara Mooney, Queensland University of Technology, Brisbane, Australia

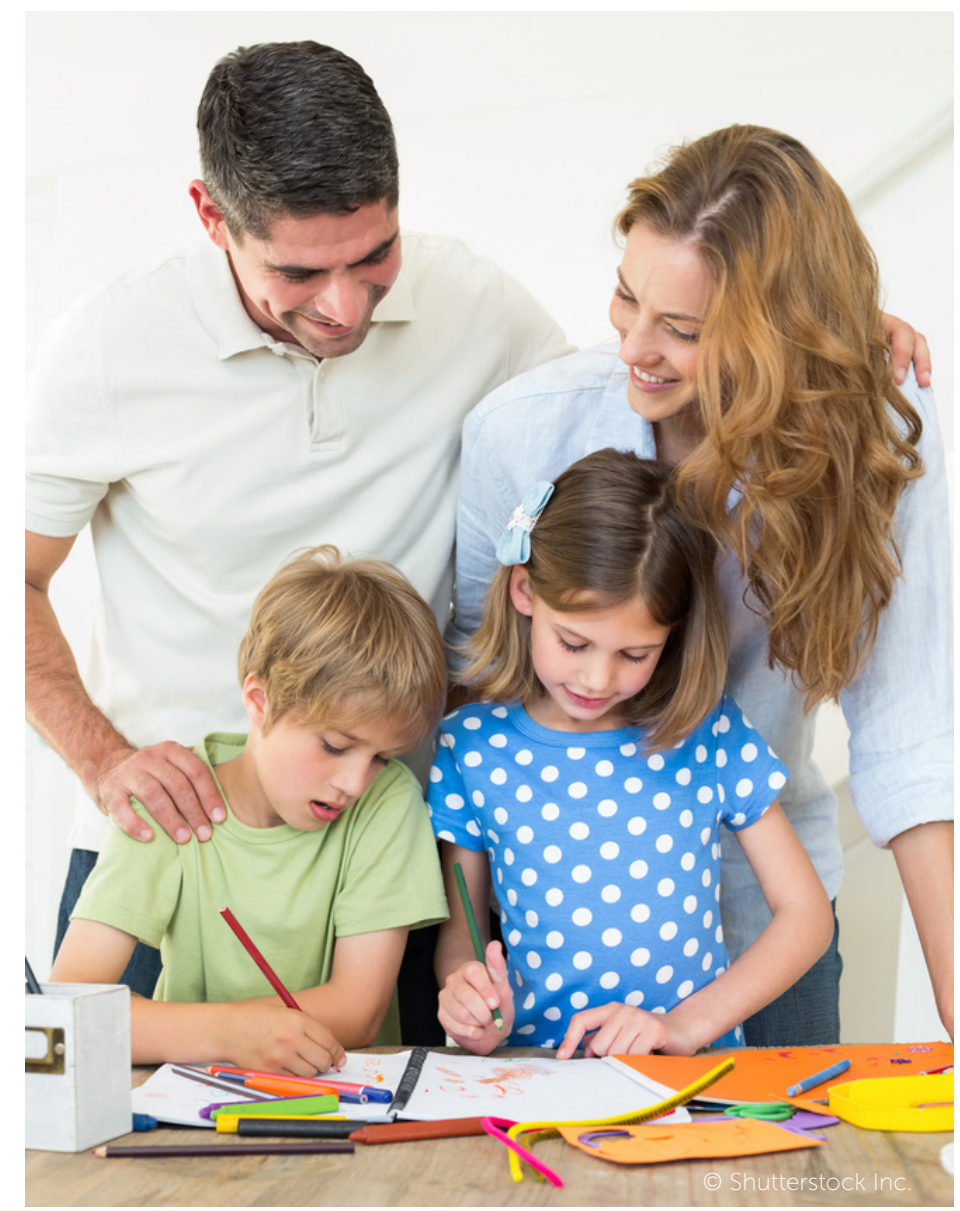

in the health care of their child with haemophilia.

The impact of haemophilia on families is associated with deterioration in the social and family relationships, which are a key factor in coping and wellbeing $[6,78]$. Parents face many hardships when managing haemophilia, including financial strains, and the cancellation or postponement of family and social plans, as well as needing to be continually prepared for bleeds, both physically and mentally [3]. Parents need to spend substantial time learning about haemophilia, including its hereditary underpinnings, and management of the condition. This can be particularly overwhelming for families who are still processing the diagnosis $[9,10]$. Parents are also faced with the challenge of trying to provide their child with normal life experiences whilst simultaneously minimising risk from harmful bleeds [11]. The desire to protect the child and provide a normal life experience is complicated by the need for treatment, 
which itself may cause the child discomfort or pain $[9,12]$. Unsurprisingly, parents often experience guilt, depression and anxiety $[3,7,13,14,15]$.

Another stressor for the parent is lack of understanding about haemophilia and management of the condition in the community, particularly amongst the child's school peers and in the parent's workplace, but also extending to the medical profession $[16,17,18,19]$. Parents have expressed a preference for seeking treatment from a specialist due to the lack of understanding about haemophilia amongst general medical professionals [16]. At the same time, the increasing shift towards home-based treatment has resulted in parents taking on responsibilities originally borne by health professionals [13]. As such, the demands upon parents for managing moderate and severe haemophilia are high [13,15].

The Australian Rural, Remote and Metropolitan (RRMA) Classification defines a metropolitan area as a capital city or city with a population greater than 100000 residents [20]. In Australia, specialist haemophilia centres or outreach services are only located in metropolitan areas. As such, living in a non-metropolitan area or some distance from specialist medical treatment creates additional challenges for persons living with haemophilia. Parents living at a distance from a haemophilia treatment centre face challenges regarding the availability of specialists and factor concentrate [21]. Difficulties associated with the acceptance of haemophilia at rural schools and access to health care services have been reported previously [18]. Proximity to a haemophilia treatment centre has been reported to have influenced decisions around pregnancy in carriers or mothers living in a family with severe haemophilia [22].

The experience of parenting a child with haemophilia, especially for parents living in non-metropolitan areas, remains relatively unknown. A number of qualitative studies have attempted to explore the experiences associated with haemophilia $[8,10,16,18,21,23]$, but few have specifically attempted to capture the experience of parenting a child with haemophilia [see 9,13]. The study by Beeton et al. [9] included both single mothers and couples; the experience of single mothers in parenting a child with haemophilia is likely to be significantly different to that of a couple. Shaw and Riley's [13] sample consisted of three different treatment modalities (home-based prophylaxis, hospital and home-based ondemand), and differences in severity and diagnosis (e.g. four children had von Willebrand Syndrome). The reduced homogeneity of these samples makes interpretation of the findings more difficult. Neither study specifically provided an understanding of the experience of parents living in non-metropolitan areas. Consequently, further qualitative research is warranted to address some of the limitations identified above and to explore the experience of parents living in non-metropolitan areas.

Interpretative phenomenological analysis (IPA) is an inductive, qualitative research method commonly utilised in Health Psychology [24]. The purpose of IPA is to gain a detailed insight into an individual's 'personal lived experience' by making sense of people's beliefs, motivations and behaviours through a process of interpretation and meaning-making $[25,26,27,28]$.

Accordingly, the aim of the following study is to employ IPA to gain an understanding of the experiences of parenting a child with moderate to severe haemophilia whilst living in a non-metropolitan area of Australia.

\section{Method - Participants}

The participants consisted of six mothers and one father who was a partner of one of the mothers included in the study. Other fathers were invited to participate but chose not to. All participants were biological parents of a child with haemophilia and living with the child. The parents' ages ranged from 25 to 48 years, while the age of their child with haemophilia ranged from 1.5 to 15 years. All seven parents were married and none had any significant health problems themselves. In terms of the highest level of education attained, two parents had completed high school, two completed technical college, one completed an undergraduate degree and one completed a postgraduate degree. In terms of employment status, one parent worked full time, one worked casually and four were homemakers. Five participants identified themselves as Australian, and one as Australian-Swedish. One participant had two children with haemophilia, and two had a known family history of haemophilia. All children had been diagnosed with haemophilia A or B within their first three years of life.

Participants were asked to complete a demographics questionnaire and the Depression Anxiety and Stress Scale (DASS-21) [29] to assess current emotional functioning for descriptive purposes. Four participants reported a normal range for anxiety, stress and depression, one rated herself as mildly depressed, and another rated herself as having severe stress and mild depression. The distance between the home of the participants and the treating haemophilia centre ranged from $100 \mathrm{~km}$ to nearly 1,000 $\mathrm{km}$. The size of the townships in which the participants lived ranged from less than 2,000 people to under 75,000 people.

\section{Procedure}

Permission to conduct the research was granted by hospital and university Human Research Ethics Committees. One of the authors (SB) discussed the project with potential participants attending or in telephone contact with the Haemophilia Treatment Centre in Brisbane. A participant information and consent form was mailed to parents. Consenting participants completed the demographics questionnaire and DASS-21 prior to the interview. The information collected was not used in any data analysis but to describe the sample. 
A semi-structured interview was developed by one of the authors (TM) based upon published recommendations [28], and used to guide an in-depth exploration of the meaning that parents assigned to the experience of parenting a child with haemophilia and their experience of the treatment regime. The interviewer asked questions around two broad themes. The first question asked was, "Tell me about your experiences of being a parent of a child with haemophilia?"

A further subset of questions was asked in order to prompt the parent to consider key periods of the development of the child, including:

- experiences when the child was first diagnosed;

- experiences when receiving medical treatment/ emergency presentations;

- experiences when the child first attended childcare and school.

The second main question asked, "Tell me about the experiences of administering the medical regime?" with follow up questions including:

- What is the experience like for you?

- How does it influence your relationships with family and friends?

- How do you experience the medical profession?

- What do these experiences mean to you?

The questions remained the same for all participants.

One participant was interviewed by telephone due to challenges in attending face-to-face, while the other participants were interviewed face-to-face at either the hospital's Haemophilia Treatment Centre or the university's Psychology Clinic. The interviews lasted between 45 minutes and two hours. All interviews were audio-recorded with the participants' permission for later transcription.

\section{Data analysis and interpretation}

The interviews were transcribed and analysed by the first author (TL) with consultation from the second and third authors (ES and SB). The interviews were transcribed verbatim and Atlas.ti 6.2 software was used to assist with the analysis process. The interview transcripts were analysed using Interpretative Phenomenological Analysis (IPA) using the procedure outlined by Smith and Osborne [28. The first interview transcript was analysed and dialogue coded to devise an initial set of themes; the other interview transcripts were then analysed sequentially. New themes were added and old themes consolidated, amended or deleted.

A process of continual reflection and interpretation of the transcripts was employed by the first author (TL), which involved re-reading and reflexive thinking. The second and third authors (EB and SB) regularly reviewed themes to improve reflexivity, to reduce interpretation bias and to ensure the interpretations reflected the participant's dialogue [28]. Memoing was used by TL during interpretation and formation of codes, and paper records of the evolution of themes and forming of master themes were maintained. The final main themes emerging from the analysis, which are presented in the following section, are supported with extracts from the interviews to allow for reader evaluation of the interpretation.

\section{Results}

Four key themes emerged from the analysis, which encapsulated the experience of those parenting a child with haemophilia and living in a non-metropolitan area:

- Bearing the brunt of diagnosis

- If you can't help me, who can?

- Tackling the challenges of treatment

- I need you to understand.

\section{Bearing the brunt of diagnosis}

The first theme illustrates the parents' strive towards acceptance of the condition, insecurities, sense of responsibility and worries as they comprehend the meaning of the diagnosis. Although the diagnosis brought a sense of relief for some parents, who now had a reason and a name for unexplained bruises, there was still uncertainty about the diagnosis and the meaning it had for their child, their family and their life. During this stage, parents had many questions and found information about haemophilia helpful, but uncertainties about haemophilia seemed to continue throughout the child's development: "it felt like we were out of control. We had this child that had something wrong with him... and how are we going to proceed with that and how was it going to affect our life and how it will affect his life?"

Mothers carry the burden of diagnosis heavily and have to process the heritable nature of the illness. This was overwhelming to those who were unaware of any family history of haemophilia. Two of the participants knew their carrier status prior to the diagnosis of their child. It appeared that prior knowledge prepared the participant and so they experienced less shock than those who did not know their carrier status. A feeling of guilt was experienced amongst the mothers interviewed, and for some guilt was something they continued to experience. Feelings of guilt stemmed from the heritable nature of haemophilia and feeling responsible for the child inheriting the disorder: "I put off a long time the thought, that, you know, we found out that it's a thing passed down through women ... the fact that, you know, I had given it to him. That was a really awful thought."

The child's attitude towards treatment had an effect on the mother's emotional functioning. If the child reacted well to treatment, the mother would report a more positive experience and seemed to cope better with the feeling of guilt. However, mothers had more difficulty coping when the child's reaction to the treatment was negative. Not being able to protect their child from pain was a cause of anguish, particularly due to the sense of responsibility the mothers felt for causing the child's distress: 
Participant: "I'd be in tears, um, even watching. I'd have to leave the room, um, because I was a nervous wreck."

Interviewer: "And, what do you think that was about? That nervousness and that, you know, distress for you, about?"

Participant: "I suppose because they've got haemophilia because of me, really. They got it no other way ... so some days I say 'Punch me,' you know, 'Give me a punch.'"

The burden of guilt experienced by the parents influenced their desire and decision to have more children. This decision was fraught with problems around the impact that potentially having a child who is a carrier or another child with haemophilia would have on themselves and their family. In this study, we assessed the total number of children in the family (between two to three) and not how many children were born after the child with haemophilia. It was therefore unclear as to whether the burden of guilt influenced only the parents' thoughts about having more children or their behaviour.

Concerns for the child's quality of life and a desire for the child to have a normal life were emphasised. The parents reported having to face continual challenges in order to provide their child with a normal life. One mother spoke about her son not being able to attend school camp because the school would not allow him to go, and another mother emphasised difficulties with her son having sleepovers.

The father expressed concerns regarding his ability to bond with his child through shared interests. Concerns were expressed around how a close relationship could develop with a son who was not able to participate in activities the father identified with. Both mothers and fathers shared disappointment around the limitations that the condition imposes and experiences the child will miss as a result: "My husband, he rides, like, snowboards and mountain bikes... that's what he has done for years and years, and to find out that [my son] can't have a skate ... that kind of door is closed for [him]. It's sad."

For some parents, haemophilia's tainted history with HIV and hepatitis infections raised concerns regarding discrimination of their child. Only one parent had a brother with haemophilia and was aware of the HIV/hepatitis stigma associated with treatment from personal experience (her brother did not have HIV). Some of the other parents were familiar with the HIV/hepatitis stigma from non-personal experiences and were concerned about their child being stigmatised.

Most parents emphasised the need to normalise the child's life and for the child not be known by their condition: "I'd like him to be [child's name], like singer, songwriter, Rhode

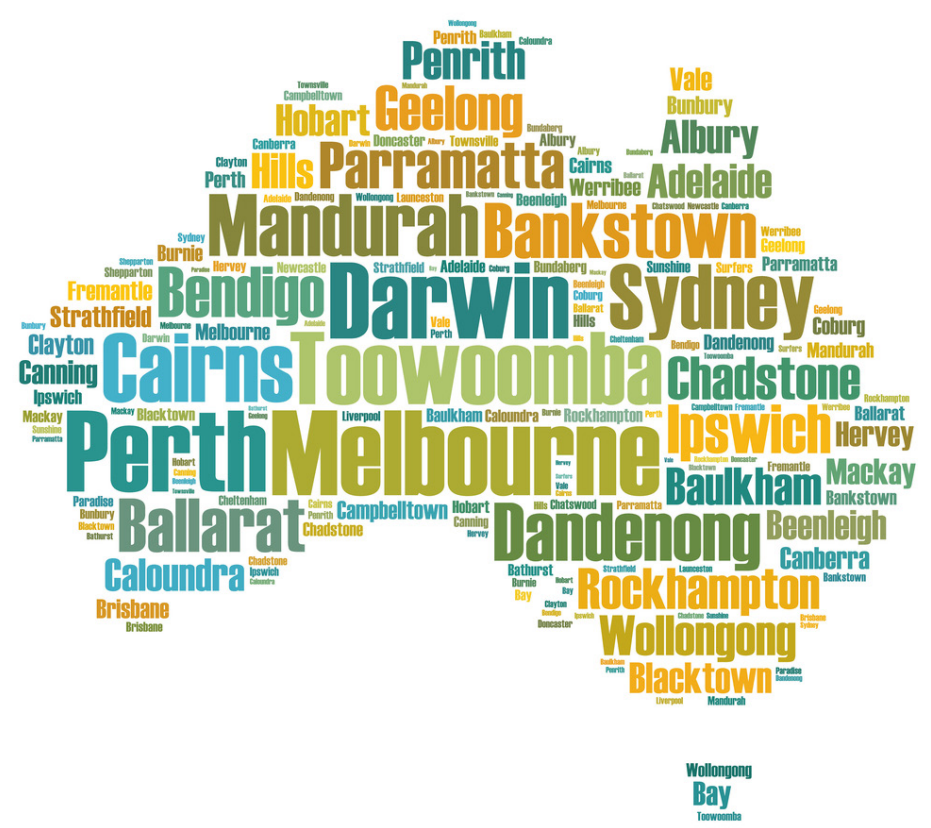

scholar ... Who has haemophilia ... rather than be haemophilia sufferer [child's name]."

Concerns were expressed by some parents about the impact of haemophilia on their child's emotional well-being. One mother was able to empathise with the difficulties her son faces and the challenges he will continue to face with managing this lifelong disorder: "It doesn't ever go away and it's every second day when that needle comes out, he is confronted by the fact that he is a haemophiliac and there is no likely change to this current situation that we can see in the foreseeable future."

In the initial weeks and months following diagnosis, the use of denial or avoidance of acknowledging haemophilia was common amongst most of the parents:

Participant: "I had a lot of denial about talking about it to other people"

Interviewer: "What do you think that would've meant, you know, if you had of spoken about it with other people?

Participant: "I would've actually had to admit that, it was real; step out of this little bubble that we had made for ourselves.

Reaching a state of acceptance reflected the parents' adjustment to the diagnosis: "we just have to look at it as being a different way of living". Reaching acceptance signified an improved capacity to cope with the challenges and burden of haemophilia. The parents expressed that over time, with increased understanding about haemophilia and practice-administering treatment, coping and living with haemophilia became easier.

\section{If you can't help me, who can?}

The second theme summarises the experience of parents 
with the health care system, illustrating the difficulties and frustrations, and desires for better access to haemophilia specialists. The first point of contact in a haemophilia emergency for many of the parents was their local general practitioner (GP) or local hospitals, many of which did not have an on-site haemophilia specialist. Difficulties involved stressful and lengthy emergency presentations and consultations with GPs or paediatricians who had limited understanding of haemophilia. The parents often described their contact with the general health care system as an unpleasant and distressing experience. "[My son] had a lot of bleeds and we had to present to the emergency department, which in itself is the most stressful, awful thing to go through; um, cause you don't get the same doctor and you can get a doctor that doesn't know about haemophilia."

Some parents experienced judgment around unexplained bruises, leaving them feeling persecuted and paranoid. Parents expressed that they felt misunderstood and ignored by the medical professionals and often faced the difficult task of trying to communicate their child's treatment needs. They also expressed discomfort and frustration around being the expert themselves, due to negative reactions from medical professionals. Consequently, such experiences lead parents to question the need to have their child treated. "We'd doubt whether we needed to go [to the hospital], because you get that 'you don't need to be here, what are you talking about?' you know, you're some schizo mother who is overreacting in emergency; that runs through your mind."

A common frustration expressed by the parents was around limited access to or availability of specialists. Most of those interviewed, expressed a desire to be located closer to the metropolitan treatment centre. "We were there for three hours because they were so unsure of what to do and then they have to ring the haematology unit in [the city] ... That was really frustrating, because if we were in [the city] we could have presented at the haematology unit."

Parents expressed concerns around the availability of and access to factor at general hospitals. For some parents, the lack of access to specialist care and limited understanding in the general medical profession translated into an inability to help: "My husband took him to the doctor and the doctor basically said, 'You need to drive another 200 kilometres because I don't know how to treat him."

It was important for parents to have access to a haemophilia specialist, even if access was via telephone contact. Although phone contact was perceived as positive support, the desire for proximity to specialists remained.

\section{Tackling the challenges of treatment}

The third theme encompasses the difficulties of managing haemophilia, including emotional difficulties associated with the parents causing harm to their child, the ability to administer treatment, feeling overwhelmed by the demands of treatment, and the sense of losing and regaining control. The parents collectively described the management of haemophilia as exhausting, challenging and an inconvenience.

The demands of treatment were incredibly consuming for some parents, who described it as the "focus" or as "dominating the landscape of the home". One parent of a child with severe haemophilia described how unyielding and emotionally draining the management of haemophilia could be: "it has been, um, 24-7, daily, weekly, monthly, yearly grind of ahhh, continual medical intervention and emotional, emotionally draining, and I think probably a grinding, wearying experience ... I think sometimes I get very down about the constant grind of it."

The parents expressed difficulties in trying to manage haemophilia and maintain other roles in their lives. They faced many logistical challenges with regard to coordinating treatment around their availability, their ability to respond to emergencies and ensuring access to factor concentrate. Nearly half of the parents (three of seven) interviewed were not working in order to be available to administer treatment or take their child to hospital for treatment.

The stress of managing their child's haemophilia was described in terms of the parents "feeling shattered" or becoming unwell and run down. Changing to prophylactic treatment reduced the stress involved in regular and often time-consuming hospital visits, but added pressures around administration. "It was hard because (pauses), you're trying to concentrate on what you are doing, making sure you're not putting air bubbles in, not touching stuff and trying to keep it sterile."

Most of the mothers viewed the treatment as painful and as a negative experience for their child, describing events surrounding treatment as traumatic. The parents who administered factor expressed how unnatural injecting their child felt, and had concerns about harming their child. Previous experience with injecting, including experiences outside of haemophilia, reduced these concerns. Any ambivalence towards treatment was lessened by the positive effect treatment had on their child as expressed by one parent: "he gets the treatment, suddenly you see him getting better, you think, oh gee, you know, it's got to be done, you know, it makes him better, it's got to be done."

Treatment challenges seemed greater during the initial stages following diagnosis or following changes in treatment type. Self-efficacy is a person's belief about their ability to successfully complete a task or achieve something. Parents reported more confidence in their ability over time: "those times when we've been unsure have lessened over the years as we've become more experienced." 
Throughout the interviews, parents frequently expressed feelings of helplessness and attempts to regain a sense of control. The unpredictably of bleeds was associated with feelings of anxiety: "The thing that really upset me was the kind of panic of not knowing when he [was] going to need treatment."

Parents would become overprotective in their parenting in order to regain some control. Some parents reported feeling powerless to prevent injury as their child became mobile. Prophylactic treatment seemed to reduce the sense of helplessness and anxiety associated with the unpredictability of bleeds and mostly had a positive effect on treatment management. With prophylaxis, haemophilia was less imposing on the parents' life, provided them with more comfort that their child would be safe from dangerous bleeds, and increased their sense of control over haemophilia: "It means we are in control ... It's a condition that we monitor now. It's not our entire life revolved around this, (pauses), thing, basically."

\section{I need you to understand}

The final theme represents the parents' desire for the understanding of others with regard to the challenges and complexities involved in caring for a child with haemophilia, and the importance of social connections and medical support in facilitating coping and well-being.

Despite having good social networks, some parents felt others did not understand the depth of the difficulties and responsibilities they had to face on a daily basis: "I guess it's an invisible responsibility, because people don't see it and even my close friends don't get it."

The need to increase others' understanding about haemophilia was important to parents, particularly within their close family and friendship circles, as well as the child's childcare and school. The need to increase understanding seemed to be driven by both a need to keep the child safe from harm and also a need for others' understanding of their experience of managing haemophilia. As emphasised below, there was comfort in the empathy of others. "They have this far-off perception that it's just an easy straightforward process ... it was great for them to see. They were far more appreciative of what we had to go through, which makes me feel a bit better."

Some parents were active in seeking connection with others in similar situations or joining the Haemophilia Foundation of Australia. They emphasised the need for advocacy and the importance of having a shared connection in instilling hope and confidence: [talking to other parents] "does make a lot of difference; you can see that someone else has got the same position as you and they're OK at the other end."

Parents expressed the need for support and the positive impact that good support had on their well-being. This included support from partners: "if he wasn't the supportive, in charge, confident person that he is about it, I think I'd be a disaster"; good medical support: "if you didn't have people that were helpful ... able to get the information to us and reassure us, I think ... the experience would have been a lot different, you know. I don't think we would have managed quiet as well"; and supportive institutions (e.g. schools, childcare and workplaces): "it's just so lovely that they're a caring and proactive school."

The parents gained comfort from having confidence and trust in the ability of others to care for their child. Without this it was difficult for parents to receive the benefits of support.

\section{Discussion}

Using interpretative phenomenological analysis (IPA), this study explored the experiences of seven parents of children with haemophilia living in a non-metropolitan area. Across each of the four main themes that emerged - bearing the brunt of diagnosis, 'If you can't help me, who can?', tackling the challenge of treatment, and 'I need you to understand' - there was an emphasis on the desire to have access to specialists, which seemed to reflect an underlying need to be heard, understood and reassured.

\section{Bearing the brunt of diagnosis}

The role of the parent is imperative in the successful management of their child's haemophilia, and parents endure the burden of their child illness. The brunt of diagnosis is experienced through the meaning parents attribute to it, and how they interpret the impact on their child and their future. Previous research has reported that parents commonly experience uncertainty at time of diagnosis and around management issues $[3,6,7]$. Similarly, previous research supports the finding that mothers carry a heavy burden of responsibility around the transmission of haemophilia, and that this is often associated with strong feelings of guilt [7].

Ensuring quality of life and normality for their child with haemophilia was important for the parents in this study. Similar findings were reported in previous qualitative studies $[9,12,13]$, reflecting the importance to parents living in both metropolitan and non-metropolitan areas of providing their child with normal life experiences. Parents in this study found comfort in the knowledge that their child was having normal life experiences.

Concerns around the ability to bond with their child with haemophilia have also been previously reported as a problem for fathers $[7,11]$. Concerns around attachment were reflected by the father in this study and through the mothers' secondary accounts of their partner's experience. Fathers seemed to struggle in particular with the physical limitations imposed by the condition, e.g. participation in various sporting activities. Parents eventually seem to reach 
a point of acceptance, where they have come to terms with the imposition haemophilia has on the child and the family's life; this was associated with better functioning and improved self-efficacy. Acceptance of haemophilia and treatment of the condition have previously been associated with improved treatment adherence [30]. The acceptance of haemophilia by the child was considered important to some mothers, who were particularly concerned about the impact of haemophilia on their child's emotional wellbeing.

\section{If you can't help me, who can?}

Difficulties associated with a lack of awareness and understanding of haemophilia amongst medical professionals, and limited access to or availability of haemophilia specialists was emphasised by the parents in the study. Interestingly, this was not the case in the study by Mooney et al. of participants living in metropolitan areas, who emphasised more appreciation of and confidence in the medical expertise and services received [12]. The main difference between these two samples was access to haemophilia specialists: parents living in proximity to the haemophilia treatment centre tended to express appreciation for the specialist medical services [12], while those parents living in non-metropolitan areas tended to express dissatisfaction with local general medical services. Access to specialist care is an important factor in the experience of parents of a child or children with haemophilia, and this finding likely reflects the specialists' understanding of haemophilia in comparison to the lack of understanding amongst the general medical profession.

Lengthy and stressful hospital admissions and feeling ignored by medical professionals resulted in most of the parents reporting negative thoughts and feelings about local hospital admissions. Negative experiences and a lack of trusting and respectful patient-provider relationships have been associated with treatment non-adherence [21,31]. Due to the difficulties experienced with general medical care, most of the parents in this study expressed a desire to have more access to or live closer to the haemophilia treatment centre. This reflected their frustrations with the limited understanding of haemophilia in the general medical profession, and their own need for reassurance and understanding. In addition, the parents in the current study expressed low confidence in the ability of local health care providers to help. This may increase the risk of injury to the child if parents either do not take their child to the local practitioner or hospital to seek local help when needed, or prolong access to treatment by driving to the metropolitan treatment centre instead.

\section{Tackling the challenges of treatment}

The parents' portrayal of the treatment regime reflected the demanding nature of haemophilia management described in previous research $[4,13,15]$. The degree to which treatment was perceived as demanding depended on the severity of haemophilia and the regularity of bleeds experienced by the child. Changing to prophylactic treatment reduced the stress involved in regular and often time-consuming hospital visits, but added pressures associated with increased responsibility for the child's treatment. This is consistent with findings in the study by Shaw and Riley [13]. Demanding management regimes have been associated with increases in depression and strained marital relationships [14]. In the present study, one parent reported marital discord, considerable stress and mild levels depression; another reported a mild level of depression. This may reflect the impact that management of their child's condition is having on their emotional functioning.

The parents perceived treatment as painful and felt that treatment was a negative experience for their child. This is consistent with previous quality of life research, where parents reported their child's experiences more negatively than the child [3]. Parents tended to express ambivalent feelings about treatment when the child reacted with distress or avoidance. This may reflect, for the mothers in particular, feelings of guilt about transmission. Ambivalence was reduced and willingness to adhere to treatment increased when parents witnessed their child improve with the treatment.

Supporting previous research findings [7, 13], prophylactic treatment was perceived as positive by the parents in this study, who felt that it gave them more control over the condition. The sense of regaining control seemed to reduce feelings of helplessness and ease anxieties associated with the unpredictability of bleeds.

\section{I need you to understand}

The role of social support in coping and adherence is reflected in the literature $[9,13,31]$. Feeling isolated was a common experience for parents living in non-metropolitan areas. On one hand, these feelings were associated with distance to medical support; on the other, parents felt that others did not understand their experience and the daily challenges they faced. The parents sought connection through shared experiences or attempts to increase the understanding of close friends and family members. The latter seemed to also be motivated by the importance of having trust and confidence in support networks, especially amongst those who play a role in caring for the child (e.g. institutions, grandparents). Access to medical support was especially important, and support in the form of phone contact was particularly helpful for parents in reducing feelings of isolation.

\section{Limitations}

There are some methodological issues for consideration. This study involved multiple researchers comparing interpretation of codes to themes rather than interpretation of data to codes. The involvement of two researchers coding that data separately would enhance the validity of findings by further reducing the influence of interpretation bias [27]. 
Another limitation was that participants were selfselected. As such, the findingsw may be biased towards parents who were more motivated, educated and better connected with haemophilia services. In addition, while all of the participants lived outside of the main metropolitan region (and therefore did not have close access to a haemophilia treatment centre), the sample did not include any participants living in remote areas. It would be interesting for a future study to explore the experiences of such participants.

Finally, the understanding of experiences and challenges with treatment would have been enhanced if a mixed method design had been used with a larger sample size, alongside the use of an objective measure of adherence to gauge which parents were high and low on adherence.

\section{Clinical implications and future research}

The findings of this study may assist clinicians and researchers in a number of ways. Asking questions based around the four themes that emerged may enable clinicians and researchers assessing parents to gain a better understanding their experiences. These themes could also be included in the development of future surveys in order to test their generalisability. In addition, quantitative research could explore the association between the themes and dependent variables, such as adherence or distress. Finally, the four themes may guide the development of interventions to promote treatment adherence and emotional functioning in parents of children with haemophilia.

\section{Conclusion}

The purpose of the present study was to gain an understanding of the experience of living in a nonmetropolitan area and parenting a child with haemophilia. Through the use of IPA, an inductive qualitative research method, the study aimed to achieve a detailed understanding of the parents' 'personal lived experience' and the meaning they make from their experiences of parenting a child with haemophilia.

Thestudy providedimportantinsightsintotheexperiences and challenges faced by parents, exemplifying the importance of access to specialist haemophilia care and the need for improved awareness of haemophilia among the general medical health profession. Four main themes emerged which reflected the meaningful experiences expressed by parents of children with haemophilia who live in non-metropolitan areas in Australia: 'bearing the brunt of diagnosis' captures the impact of the diagnosis; 'If you can't help me, who can?' reveals experiences with the health system; 'tackling the challenge of treatment' encompasses difficulties in adhering to the treatment regime; and 'I need you to understand' reflects desire for the understanding and support of others. These four themes may help guide researchers and clinicians in supporting parents of children who live in nonmetropolitan areas and do not have ready access to haemophilia treatment centres.

\section{Disclosures}

The authors have advised that there are no interests that might be perceived as posing a conflict or bias.

This is an Open Access article distributed under the terms of the Creative Commons Attribution License (http://creativecommons.org/ licenses/by/2.0), which permits unrestricted use, distribution, and reproduction in any medium, provided the original work is properly cited.

\section{References}

1. Haemophilia Foundation Australia. Haemophilia. 2009. Victoria: Haemophilia Foundation Australia.

2. Manco-Johnson MJ, Riske B, Kasper CK. Advances in care of children with hemophilia. Seminars in Thrombosis and Hemostasis 2003; 29: 585-94.

3. Banis $S$, Suurmeijer $T$, van Peer DR. Child-rearing practices toward children with hemophilia: The relative importance of clinical characteristics and parental emotional reactions. Family Relations 1999; 48: 207-13.

4. Thornburg CD. Prophylactic factor infusions for patients with hemophilia: Challenges with treatment adherence. Journal of Coagulation Disorders 2009; 2(1): 9-14.

5. Ljung RC. Intracranial haemorrhage in haemophilia A and $\mathrm{B} . \mathrm{Br} \mathrm{J}$ Haematol 2008; 140 (4):378-84.

6. Coppola A, Cerbone AM, Mancuso G, et al. Confronting the psychological burden of haemophilia. Haemophilia 2011; 17: 21-7.

7. Saviolo-Negrin N, Cristante F, Zanon E, et al. Psychological aspects and coping of parents with a haemophilic child: a quantitative approach. Haemophilia 1999; 5: 63-8.

8. Thomas S, Herbert D, Street A, et al. Attitudes towards and beliefs about genetic testing in the haemophilia community: a qualitative study. Haemophilia 2007; 13: 633-41.

9. Beeton K, Neal D, Watson T, et al. Parents of children with haemophilia - a transforming experience. Haemophilia 2007; 13: 570-9.

10. Gregory $M$, Boddington $P$, Dimond $R$, et al. Communicating about haemophilia within the family: the importance of context and of experience. Haemophilia 2007; 13: 189-98.

11. Wiedebusch S, Pollmann H, Siegmund B, et al. Quality of life, psychosocial strains and coping in parents of children with haemophilia. Haemophilia 2008; 14: 1014-22.

12. Mooney TS, Strodl E, Brown SA. Mothers of children with haemophilia: An exploration of their experiences. Brisbane: Queensland University of Technology, 2012

13. Shaw D, Riley GA. The impact on parents of developments in the care of children with bleeding disorders. Haemophilia 2008; 14: 65-7. 14. Steele RG, Forehand R, Armistead L. The role of family processes and coping strategies in the relationship between parental chronic illness and childhood internalising problems. Journal of Abnormal Child Psychology 1997; 25: 83-94.

15. Teitel JM, Barnard D, Israels $S$, et al. Home management of haemophilia. Haemophilia 2004; 10: 118-33.

16. Barlow JH, Stapley J, Ellard DR. Living with haemophilia and von Willebrand's: A descriptive qualitative study. Patient Education and Counselling 2007; 68: 235-42.

17. George A, Vickers MH, Wilkes L, et al. Working and caring for a child with chronic illness: Barriers in achieving work-family balance. Journal of Management \& Organization 2008; 14: 59-72.

18. Talaulikar D, Shadbolt B, McDonald A, et al. Health-related quality of life in chronic coagulation disorders. Haemophilia 2006; 12: 633-42. 19. Williams KA, Chapman MV. Social challenges for children with 
hemophilia: Child and parent perspectives. Social Work in Health Care 2001; 50: 199-214.

20. Australian Institute of Health and Wellfare. Rural, Remote and Metropolitan Areas (RRMA) Classification. [n.d.] Available from http://www.aihw.gov.au/ rural-health-rrma-classification (accessed 17 November 2015).

21. Pritchard AM, Reimer M, Simonson K, et al. Partnerships in specialty care: Exploring rural haemophilia provider resource needs. Australian Journal of Rural Health 2006; 14: 184-9.

22. Kadir RA, Sabin CA, Goldman E, et al. Reproductive choices of women in families with haemophilia. Haemophilia 2000; 6: 33-40.

23. Gannoni A, Shute R. Parental and child perspectives on adaptation to childhood chronic illness: A qualitative study. Clinical Child Psychology and Psychiatry 2010; 15: 39-53.

24. Smith JA. Beyond the divide between cognition and discourse: Using interpretative phenomenological analysis in health psychology. Psychology $\&$ Health 1996; 11: 261-71.

25. Eatough V, Smith J. 'I was like a wild wild person': Understanding feelings of anger using interpretative phenomenological analysis. British Journal of Psychology 2006; 97: 483-98.

26. Larkin M, Watts S, Clifton E. Giving voice and making sense in interpretative phenomenological analysis. Qualitative Research in Psychology 2006; 3: 10220

27. Smith JA. Reflecting on the development of interpretative phenomenological analysis and its contribution to qualitative research in psychology. Qualitative Research in Psychology 2004; 1: 39-54.

28. Smith JA, Osborn M. Interpretative phenomenological analysis. In: Smith JA (ed.) Qualitative Psychology. A Practical Guide to Research Methods, 2nd edn. London: SAGE Publications Ltd, 2008.

29. Lovibond PF, Lovibond SH. The structure of negative emotional states: Comparison of the Depression Anxiety Stress Scales (DASS) with the Beck Depression and Anxiety Inventories. Behaviour Research and Therapy 1995; 33: $335-43$.

30. Moosa MYH, Jeenah FY, Kazadi N. Treatment adherence. South African Journal of Psychiatry 2007; 13: 40-5.

31. Moerloose PD, Urbancik W, van den Berg M, Richards M. A survey of adherence to haemophilia therapy in six European countries: results and recommendations. Haemophilia 2008; 14: 931-8.

\section{The Journal of} Haemophilia Practice

\section{An open-access} journal for sharing experience in the care of

\section{people with} bleeding disorders

\section{www.haemjournal.com}

